\title{
TRANSPORT PROPERTIES OF GRAPHENE BILAYER ON SUBSTRATE FROM EXACT ELECTRONIC GREEN'S FUNCTION
}

\author{
Nadezhda G. Bobenko, Alexander N. Ponomarev, Alexander A. Reshetnyak \\ www.ispms.ru/ \\ 2/4, pr. Akademicheskii, 634021 Tomsk, Russian Federation \\ nlitvin86@rambler.ru, alex@ispms.tsc.ru, reshet@ispms.tsc.ru \\ Dalibor Čevizović \\ Vinča Institute of Nuclear Sciences, University of Belgrade, http://www.vin.bg.ac.rs/ \\ P.O.Box 522, Mike Petrovića Alasa 12-14, 11001 Belgrade, Serbia \\ cevizd@vin.bg.ac.rs
}

Institute of Strength Physics and Materials Science Siberian Branch of Russian Academy of Sciences, http://

Abstract. We consider two approaches to find both the longitudinal and Hall conductivities of ABstacked bilayer graphene in dependence of frequency, finite chemical potential, temperature with magnetic field on a base of 2- and 4-band effective models. The relations to be important for optoelectronic among Hall conductivities and Faraday, Kerr angles in the AB-bilayers samples in the electric and magnetic fields when the radiation passes across bilayer sheets on different kinds of substrate are derived. We obtain the low-temperature electrical resistivity (conductivity) of the epitaxial graphene on a base of the temperature electron Green functions method adopted for carbon nanosystems from metallic system. The calculations of contribution to the conductivity (in addition to Drude part) is realized with account for multiple elastic scattering of electrons by impurities and structural inhomogeneities of short-range order.

Keywords: graphene, band effective model, conductivity, temperature electron Green functions method, elastic scattering of electrons, structural inhomogeneities

PACS: 68.65.Pq, 72.80.Vp, 75.47.-m, 73.43.-f, 72.40.+w

Bibliography - 15 references

Received 15.10.2015

RENSIT, 2015, 7(2):168-174

DOI: 10.17725/rensit.2015.07.168

CONTENTS

1. INTRODUCRION (168)

2. Models for AB BiLAYer (169)

3. Optical conductivities in and without MAGNETIC FIELD (170)

4. Density OF STATES AND RESISTIVITY FOR NONIDEAL GRAPHENE (171)

5. Conclusion (173)

REFERENCES (173)

\section{INTRODUCTION}

$A B$ graphene bilayer [1] presents a unique sample of $2 D$ condensed matter systems possessing by outstanding transport and mechanical properties. Its low-energy electron spectrum [2, 3] combines characteristics of monolayer graphene and usual $2 D$ electron systems due to consisting of 2 inequivalent pairs of parabolic valence and conductance bands, touching each other at $K_{+}\left(K^{\prime}\right)$ and $K_{-}(K)$ Dirac points with massive charge carriers. By the main property (for optoelectronic) of the bilayer is the opening of a tunable gap between the valence and conduction bands when an electric field $\mathrm{E}$ acts on it (see, Fig. 1).

Fig. 1 shows the reciprocal lattice of bilayer graphene (left) with lattice points indicated as crosses, with $b_{1}=2 \pi / a(1,1 \sqrt{ } 3), b_{2}=2 \pi / a(1,-1 \sqrt{ } 3)$ being by primitive reciprocal lattice vectors, for lattice constant $a=2.46 \AA ; d_{0}=3.35 \AA$ (distance among adjacent unit cells and layers), the shaded hexagon appears by the first Brillouin zone with the centre $\Gamma$, and the Dirac points $\mathrm{K}_{-}\left(\mathrm{K}^{\prime}\right), \mathrm{K}_{+}(\mathrm{K})$ - showing
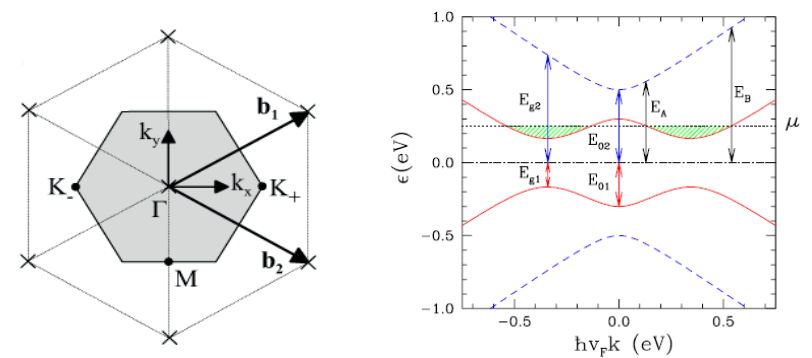

Fig. 1. Reciprocal lattice of bilayer graphene (left) and the lowenergy bands of bilayer for the first Brillowin zone (right). 
two non-equivalent corners. From the right the lowenergy bands of bilayer near K', K points around the Fermi level in the presence of the gap $\Delta$ for $\Delta=0.3$ $\mathrm{eV}, \gamma_{1}=0.4 \mathrm{eV}$ and chemical potential $\mu=0.25 \mathrm{eV}$ for the first Brillouin zone. Plots were adapted from $[9,10]$.

Note, the value of $E$ can be controlled externally by chemical doping (impurities) and gating. Since, the density of states remains finite in the unbiased and neutral bilayer, there are predictions [5] that the electron-electron interaction can result in spontaneous symmetry breaking and opening a gap for $B=0$.

The nature of the gapped state is much discussed in the literature. Possible scenarios include anomalous quantum Hall (QAH), quantum spin Hall (QSH), layer antiferromagnet (LAF) states, etc. Formally, all these gapped states are differed by the variant of breaking an approximate $S U(4)$ spin-valley symmetry of the low energy Hamiltonian of bilayer graphene.

Fig. 2 shows the view in a perpendicular direction to the bilayer sheets (top) and (b) side view of the crystal structure of bilayer graphene. Atoms $A 1$ and $B 1$ on the lower layer are shown as white and black dots, $A 2, B 2$ on the upper layer are black and grey, correspondingly. The shaded rhombus in (top) indicates the conventional unit cell with 4 atoms $A 1, B 1$ (on the lower layer), $A 2, B 2$ (on the upper layer). On the bottom the hopping parameters are presented.

The finding of a theoretical model describing an energy spectrum and (magneto)-optical conductivities of $A B$-bilayer in external electric, magnetic fields for nonvanishing temperature and
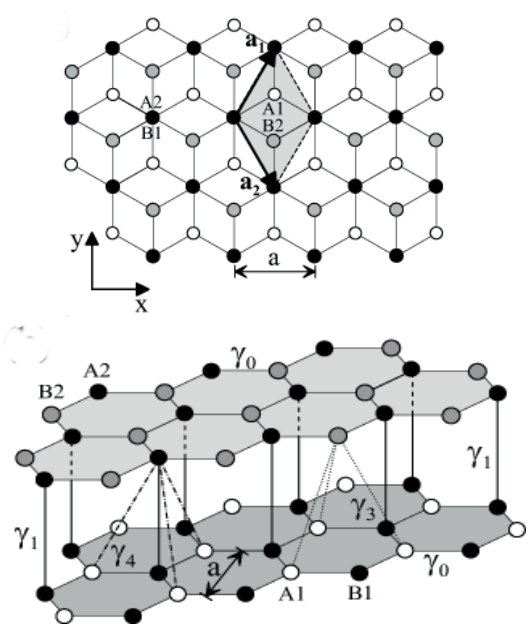

Fig. 2. View in a perpendicular direction to the bilayer sheets (top) and (b) side view of the crystal structure of bilayer graphene. densities of charged carriers is not yet completely solved (see, the results on conductivity for 4-band model [4]). In turn, the influence of the substrate on which is usually realized graphene, within epitaxial growth, leads to the appearance of the structural inhomogeneities $(\mathrm{SiH})$ which should to account for the calculation of the electric and magneto-optic conductivities (resistivity). For the latter problem we apply the temperature electron Green functions $(G F)$ method [7] adopted for $1 D, 2 D$ carbon materials [8].

The paper is organized as follows. In the Sec. 2 we calculate on a base of 2- and 4-band effective Hamiltonians the longitudinal and transverse (Hall) optical conductivities and study the choice of the different gapped states on it. The relations among the Hall conductivities and Faraday and Kerr angles when pure bilayer interacts with substrates are analyzed in the Sec. 3. Then we obtain the DOS and resistivity for monolayer with impurities interacting with substrate on a base of electron (metallic) GF.

\section{MODELS FOR AB BILAYER}

The tight-binding model developed for graphite [5, 6] and known as Slonczewski-Weiss-McClure (SWC) model are easily formulated for Hamiltonian of $A B$ stacked graphene according hopping parameters in the Fig. 2 as

$$
\begin{aligned}
& H_{t b}=-\gamma_{0} \sum_{(i, j), m, s=1,2} a_{m, i, s}^{+} b_{m, j, s}-\gamma_{4} \sum_{i ; s=1,2}\left(a_{1, i, s}^{+} a_{2, i, s}+b_{1, i, s}^{+} b_{2, i, s}\right)- \\
& -\gamma_{1} \sum_{i ; s=1,2} a_{2, i, s}^{+} b_{1, i, s}-\gamma_{3} \sum_{i ; s=1,2} a_{1, i, s}^{+} b_{2, i, s}+\text { h.c., }
\end{aligned}
$$

where $a_{m, i, s}^{+}\left(b_{m, j, s}^{+}\right)$creates an electron with spin $s=$ $-\hbar / 2, \hbar / 2$ in layer $m=1,2$ on sublattice $A(B)$ at site $\mathbf{R}_{i}$ with hopping parameters $\left(\gamma_{0}, \gamma_{1}, \gamma_{3}, \gamma_{4}\right)=(3.0,0.38$, $0.1,0.12) \mathrm{eV}$ [3].

In the continuum limit, to study the electronic properties, when expanding the momentum $p=\hbar(k$ $+K_{3}$ in the vicinity of $K, K^{\prime}$ points of fixed unit sell of the bilayer we obtain the effective 4-band Hamiltonian with 4-component spinor wave function $\Psi_{4}$, with general form for the gap containing valley and spin depending terms $[9,10]$

$$
\begin{aligned}
& H=\xi\left(\begin{array}{ll}
H_{11} & H_{12} \\
H_{12}^{+} & H_{22}
\end{array}\right), H_{11}=\left(\begin{array}{cc}
\Delta_{\xi s} & \gamma_{3} v_{F} \pi / \gamma_{0} \\
\gamma_{3} v_{F} \pi^{+} / \gamma_{0} & -\Delta_{\xi s}
\end{array}\right), \\
& H_{22}=\left(\begin{array}{cc}
-\Delta_{\xi s} & \gamma_{1} \\
\gamma_{1} & \Delta_{\xi s}
\end{array}\right), H_{12}=\left(\begin{array}{cc}
\gamma_{4} v_{F} \pi^{+} / \gamma_{0} & v_{F} \pi^{+} \\
v_{F} \pi & \gamma_{4} v_{F} \pi / \gamma_{0}
\end{array}\right)
\end{aligned}
$$
for $\Delta_{\xi \mathrm{S}}=U+\xi_{\mathrm{T}}+s U_{T}+\xi_{s} \Delta$, with Fermi velocity $\nu_{\mathrm{F}}$ $=10^{6} \mathrm{~m} / \mathrm{s}$ in plane of layers, $U, \Delta\left(U_{T}, \Delta_{T}\right)$ being by (not) invariant with respect to time reversal, whereas the first gap $U$ can be induced by a perpendicular 
electric field $E$. In this case $U=e E d_{0} / 2$ with $-e$ being by the charge of electron. The momentum $\pi=p_{x}+i_{y}$ in (2) may be enlarged by means of preserving of the $U(1)$ gauge invariance for $H$ in case of bilayer interaction with external magnetic field $\mathbf{B}$ being applied perpendicular to the sheets along the positive axis z realizing the case of QED in (2+1)-dimensional space-time to the quantity: $\hat{\pi}=\hat{p}_{x}+i \hat{p}_{y}=-i \hbar\left(D_{x}+i D_{y}\right)$ for covariant derivative $D_{i}$ $=\partial_{i}+(i e / \hbar c) A$, with vector potential $A(x, y)=(0$, $B x)$ in the Landau gauge. $H$ acts on $\Psi_{4}$ corresponding to the atomic sites $A 1, B 2, A 2, B 1$ in the valley $K$ $(\xi=+1)$ and $B 2, A 1, B 1, A 2$ in the valley $K^{\prime}(\xi=$ $-1)$. Here, we neglect the tight-binding parameters $\gamma_{3}$ (leading to a trigonal warping of the band structure at low energies and $\gamma_{4}$ ).

The role of different kinds of gaps in the magneto-transport properties may be studied for low-energy approximation, for energy range $|\epsilon|<$ $\gamma_{1} / 4$ within 2-band model with Hamiltonian:

$$
H_{e f f}=\xi\left(\begin{array}{cc}
\Delta_{\xi s} & -\xi\left(\bar{\pi}^{+}\right)^{2} / 2 m \\
-\xi \bar{\pi}^{2} / 2 m & -\Delta_{\xi s}
\end{array}\right)
$$

with effective mass $m=\gamma_{1} /\left(2 v_{F}^{2}\right)=0.032 m$, which is obtained from 4-band Hamiltonian (2). In turn, $H_{\text {eff }}$ acts on a 2-component wave function $\Psi_{2}$ corresponding to the atomic sites $A 1, B 2$ in the valley $K(\xi=+1)$ and $B 2, A 1$ in the valley $K^{\prime}(\xi=$ $-1)$.

The Hamiltonians possess by the reflection properties in depending on the form of the gap. Thus, in the $4 \times 4$ case (2) the presence of the gap $\Delta_{\xi s}$ $=\xi \Delta_{\mathrm{T}}$ breaks the time-reversal symmetry.

\section{OPTICAL CONDUCTIVITIES IN AND WITHOUT MAGNETIC FIELD}

The calculation of the optical conductivities is based on the Kubo formula,

$$
\sigma_{i j}(\Omega)=(\hbar / i \Omega)\left(\Pi_{i j}^{R}(\Omega+i 0)-\Pi_{i j}^{R}(0)\right),
$$

for $\quad \Pi_{i j}^{R}(i \Omega)=\Pi_{i j}\left(i \Omega_{m} \rightarrow \Omega+i \varepsilon\right), i, j=x, y \quad$ with retarded current-current correlation function $\Pi_{i j}^{R}(\Omega+i 0)$ obtained by the analytical continuation from its imaginary time expression with $\Omega$ being by the photon energy. The calculation of the current-current correlation function in tree level approximation reduces to the evaluation of the bubble diagram

$$
\begin{aligned}
& \Pi_{i j}\left(i \Omega_{m}\right)=-\frac{1}{V} \int_{0}^{\beta} d \tau e^{i \Omega_{m} \tau} \iint d^{2} r d^{2} r^{\prime} t r_{\xi, s} \times \\
& \times\left[j_{i}(r) G\left(r, r^{\prime}, \tau\right) j_{j}\left(r^{\prime}\right) G\left(r^{\prime}, r,-\tau\right)\right]
\end{aligned}
$$

with $j_{i}(r)=-c \partial H / \partial A^{i}$, being by the electric current density operator. The quantities $G(r, r, \tau), V, \beta=$ $1 / T, \Omega_{\mathrm{m}}=2 \pi \mathrm{m} / \beta$ are the electron $G F$, the volume of the system, the inverse temperature, and $\operatorname{tr}_{\bar{\zeta}, s}$ means for trace.

Note, the presence or absence of a magnetic field $B$ in both 2- and 4-band cases permits to use different technique due to Landau levels (LLs) in the energy spectrum for $B \neq 0$.

For the 2-band model in a magnetic field the optical conductivities $\sigma_{ \pm}(\Omega)=\sigma_{\mathrm{xx}}(\Omega) \pm i \sigma_{\mathrm{xy}}(\Omega)$ corresponding to the opposite circular polarizations of light have the form (for magnetic length $l=\sqrt{h c / e B}[9])$,

$\sigma_{ \pm}(\Omega)=e^{2} \hbar^{2} / 2 \pi m^{2} l^{4} \sum_{k \geq 0}(k+1) \times$

$\left.\times \sum_{v, v^{\prime}, \xi, s= \pm}\left\{\begin{array}{l}{\left[\frac{1}{v^{\prime} M_{k+2}-v M_{k+1}}\left(1-\frac{v v^{\prime} \xi \Delta_{\xi s}}{M_{k+1} M_{k+2}}\right) \pm \frac{v v^{\prime} \xi \Delta_{\xi s}}{M_{k+1} M_{k+2}}\right.} \\ \times \frac{i\left(n_{F}\left(v M_{k+1}\right)-n_{F}\left(v^{\prime} M_{k+2}\right)\right)}{\Omega \mp v^{\prime} M_{k+2} \pm v M_{k+1}+2 i 0}\end{array}\right\} \times\right\}$

for Fermi-Dirac distribution function $n_{F}(\omega)$, energy LLs $E_{n \xi}$ for $\Delta_{\xi}=\Delta+\xi \Delta T$ determined from the spectral problem for the stationary Schrödinger equation $H_{e f f} \Psi_{2 \mathrm{n}}=E_{n \xi} \Psi_{2 \mathrm{n}}: E_{n \xi}=-\xi \Lambda_{\xi}, \mathrm{n}=0,1 ; E_{ \pm n \xi}= \pm M_{n \xi}$. $M_{n \xi}=\sqrt{\Delta_{\varepsilon}^{2}+\omega_{c}^{2} n(n-1)}, n \geq 2$ for cyclotron energy $\omega_{c}$ [9].

The limiting cases of $B=0$ for the Hall conductivity $\sigma_{\mathrm{xy}}(\Omega)=\left(\sigma_{+}(\Omega)-\sigma_{-}(\Omega)\right) / 2 i$ and its observable real part, $\operatorname{Re} \sigma_{\mathrm{xy}}(\Omega \rightarrow 0)$, for direct current obtained from (6) for continuum limit $\left(M_{k}\right.$ $\rightarrow \omega)$ [9]

$$
\begin{aligned}
& \sigma_{x y}(\Omega, B=0)=-4 e^{2} / \hbar \sum_{\xi, s= \pm} \xi \Delta_{\xi s} \int_{\left|\Delta_{\xi s}\right|}^{\infty} d \omega \frac{\left(n_{F}(\omega)-n_{F}(-\omega)\right)}{4 \omega^{2}-(\Omega+i 0)^{2}}, \\
& \operatorname{Re} \sigma_{x y}(\Omega \rightarrow 0)=e^{2} / \hbar \sum_{\xi, s= \pm} \xi \begin{cases}\operatorname{sgn}\left(\Delta_{\xi s}\right), & |\mu|<\left|\Delta_{\xi_{s}}\right| \\
\Delta_{\xi s} /|\mu|, & |\mu| \geq\left|\Delta_{\xi s}\right|\end{cases}
\end{aligned}
$$

do not vanish for time-reversal breaking gaps $U_{T}$, $\Delta_{T}$ and for neutral point, $\mu=0$, corresponds to the quantum anomalous Hall effect in bilayer.

For the 4-band model with the Hamiltonian (2) in a magnetic field the conductivities may be determined in the same way as for 2-band model. We restrict ourselves by only Hall $\sigma_{\mathrm{xy}}(\Omega)$ conductivity: 


$$
\begin{aligned}
& \sigma_{x y}(\Omega)=e^{2} V_{F}^{2} \hbar / \pi l^{2} \sum_{k \geq 0} \times \\
& \times \sum_{\alpha, \beta, \alpha^{\prime}, \beta^{\prime}, \xi,= \pm}\left\{\begin{array}{l}
{\left[\frac{\left(n_{F}\left(E_{k+1, \alpha^{\prime}, \beta^{\prime}}(\xi)\right)-n_{F}\left(E_{k, \alpha, \beta}(\xi)\right)\right)}{E_{k, \alpha, \beta}(\xi)-E_{k+1, \alpha^{\prime}, \beta^{\prime}}(\xi)}\right] \times} \\
\times\left|\begin{array}{l}
C_{1, k}^{*}(\alpha, \beta, \xi) C_{4, k+1}\left(\alpha^{\prime}, \beta^{\prime}, \xi\right)+\left.\right|^{2} \\
+C_{3, k}^{*}(\alpha, \beta, \xi) C_{2, k+1}\left(\alpha^{\prime}, \beta^{\prime}, \xi\right)
\end{array}\right| \times \\
\times\left(\begin{array}{l}
\left(\Omega+i \Gamma+E_{k, \alpha, \beta}(\xi)-E_{k+1, \alpha^{\prime}, \beta^{\prime}}(\xi)\right)^{-1}- \\
-\left(\Omega+i \Gamma-E_{k, \alpha, \beta}(\xi)+E_{k+1, \alpha^{\prime}, \beta^{\prime}}(\xi)\right)^{-1}
\end{array}\right)
\end{array}\right\}
\end{aligned}
$$

where the constants $C_{i k}(\alpha, \beta, \xi)$ and LLs now for each $\mathrm{n}$ has 4-band, not-degenerate in spin $s$ and valley $\xi$ and determined from the exact solution for the spectral problem $H \Psi_{4 \mathrm{k}}=E_{k, a, \beta}(\xi) \Psi_{4 \mathrm{k}}\left(\mathrm{s}, \mathrm{C}_{1 \mathrm{k}}(\alpha, \beta, \xi)\right.$, $\left.C_{2 k}(\alpha, \beta, \xi), C_{3 k}(\alpha, \beta, \xi), C_{4 k}(\alpha, \beta, \xi)\right)$ in the form (for details see, [10]).

The spontaneously broken time-reversal symmetry in bilayer can be observable via optical polarization rotation when light is transmitted through the sample (Faraday effect) or reflected by it (Kerr effect) among them for: 1) free-standing bilayer with a refractive index $n=1$ (the first inset from the top in Fig. 3); 2) graphene on a thick substrate (corresponding to $\mathrm{SiO}_{2}, \mathrm{BN}$ ) with $n=1.5$; 3) for bilayer on a dielectric layer with $n=1.5$ and a thickness of $d=300 \mathrm{~nm}$ on top of a thick layer with $n_{s}=3.5$ (corresponding to the standardly used $\mathrm{SiO}_{2} /$ Si substrates). The Faraday and Kerr angles have the form:
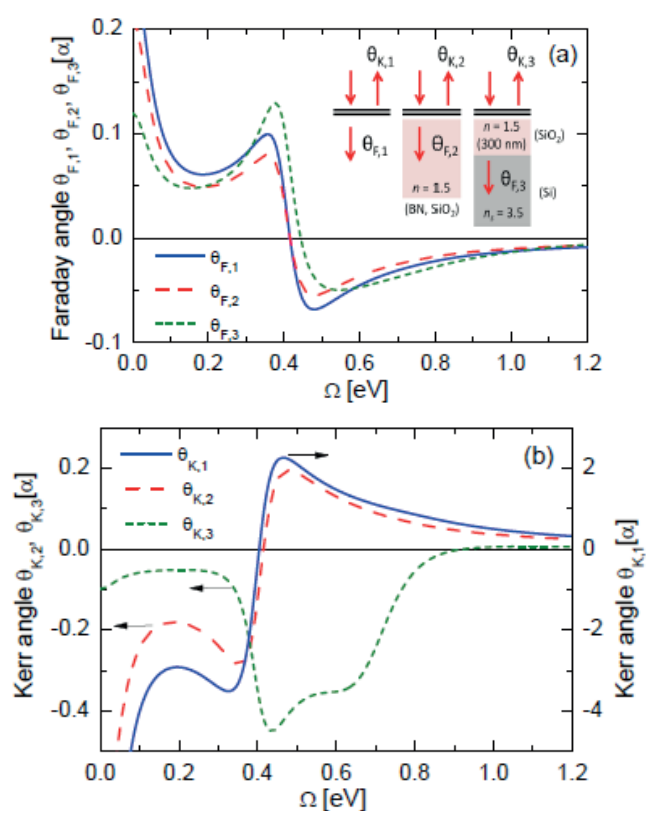

Fig. 3. The calculated Faraday (top) and Kerr (bottom) rotation angles for various experimental geometry shown in inset. $\sigma_{ \pm}(\Omega)$ calculated within 2-band model for non-vanishing time-reversal breaking gap $\Delta_{T}\left(\Delta_{T}=0.001 \mathrm{eV}\right)[9]$.

$$
\begin{aligned}
& \theta_{F}\left(\theta_{K}\right)=0.5\left(\arg \left(t_{-}\left(r_{-}\right)\right)-\arg \left(t_{+}\left(r_{+}\right)\right)\right), \\
& \left(r_{ \pm}, t_{ \pm}\right)=\left(1+n+Z_{0} \sigma_{ \pm}\right)^{-1}\left(1-n-Z_{0} \sigma_{ \pm}, 2\right)
\end{aligned}
$$

(for the reflection, transmission coefficients $r_{ \pm}, t_{ \pm}$at 'vacuum-film-substrate' interface, for $Z_{0}=4 \pi / c$ ).

The dependence of the Faraday and Kerr angles on $\Omega$ for various samples is shown on Fig. 3, where for different samples the only $\Theta_{K}$ for the third sample with double substrate has another dependence.

\section{DENSITY OF STATES AND RESISTIVITY FOR NON-IDEAL GRAPHENE}

Here, we turn to the low-temperature behavior of electrical resistivity for direct current (the quantity corresponding to $\left(\operatorname{Re} \sigma_{\mathrm{xy}}(\Omega \rightarrow 0)\right)^{-1}$ and electron DOS in non-ideal graphene. It is well known that DOS strongly depends on its "non-ideality" which is characterized by some distribution of impurities and structural defects of different types [11] due to its interaction with various kinds of substrates leading to changing of the type of conductivity (semiconductor - metal) in graphene [12].

The low-temperature peculiarities of electrical resistivity and DOS in non-ideal graphene may be obtained in a way being different to one considered above and following from the temperature GF method [7] for the electron in a metal which was used in $[8,13,14]$ to describe the low-temperature electron transport properties and DOS in non-ideal carbon nanotubes. Note, however, this approach can not provide the correct limit in case of absence of the impurities and structural defects. These peculiarities are especially revealed for room $T=300 \mathrm{~K}$ and there are no results accounting of magnetism.

To develop the last approach we use the electronic spectrum [15] for the $2 D$ monolayer. A description of the graphene as the system with impurities and $\mathrm{SiH}$ of short-range order is based on $2 D$ model of a solid material with randomly distributed defects such as catalyst impurities, retained atoms. A random field of adsorbed atoms of one sort $V(R)=\sum c\left(\vec{R}_{i}\right) U\left(\vec{R}_{i}-\vec{R}\right)$, depends on $c(R)$ and $U$ being by the occupation numbers and the potential of an electron in cite $R_{i}$ of graphene. For long-range ordering absence the representation $c(R)$ $=c+\delta c(\mathrm{R})$ allows to introduce a correlator, $<\delta c(\mathrm{R})$ $\delta c\left(R_{i}\right)>$, which determines new chemical bonds (or new short-range ordering) in a system with defects via fluctuations of concentration $\delta c(R)$ in site $R_{i}$ 
and averaged over field $V$ macroconcentration $c=$ $<c(\mathrm{R})>$. The Fourier transform $\langle\delta c(\mathrm{R}) \delta c(\mathrm{R})\rangle \sim$ $<\left|c_{k}\right|^{2}>$ determines the structure of short-range order $\left\langle\left|c_{k}\right|^{2}\right\rangle=c(1-c) / N \sum_{i=1}^{N} \alpha_{i} \cos \left(k \cdot R_{i}\right)$ with $\alpha_{\mathrm{i}}, N$ are the short-range order coefficients $\left(\alpha_{0}=1\right)$, number of atoms in $\mathrm{SiH}$.

To calculate one-particle properties, e.g. the electronic relaxation time, we present the one-particle irreducible (vertex) GF in the form (following from Dyson-Schwinger equation [7]):

$$
G=G_{0}+\sum_{i} G_{i}^{(1)}+\sum_{i j} G_{i j}^{2}+\ldots
$$

with electron $G F G_{0}=\left(\varepsilon-\varepsilon_{\mathrm{p}}+i 0\right)^{-1}$ in a metal system and $G_{i}^{(1)}=c\left(R_{i}\right) \int G_{0}(\vec{r}, R) U\left(R-R_{i}\right) G_{0}\left(R, \vec{r}^{\prime}\right) d R$. In the momentum representation we obtain the GF for a "dirty" (non-ideal) graphene: $\langle G\rangle=G_{0}+G_{0}^{2} \Sigma$, with self-energy part $\Sigma$ of $G F$, including multiple elastic scattering of the electrons on impurities and $\mathrm{SiH}$ in the form

$$
\begin{aligned}
& \Sigma(p, \varepsilon)=-2 \pi i c U_{0}^{2} v_{0} \times \\
& \times \sin \varepsilon\left(1-(1-c) N^{-1} \sum_{i=1}^{N} \alpha_{i}\left(1-R^{2} m / \hbar^{2}\left(\varepsilon+\varepsilon_{F}+i 0 \operatorname{sign} \varepsilon\right)\right)\right) .
\end{aligned}
$$

Here $U_{0}$ is the effective potential and $\nu_{0}=2 \pi \mathrm{m} / h^{2}$ is the value of DOS at the Fermi level $\varepsilon_{\mathrm{F}}$ The first and order terms correspond to the contribution from electron scattering on impurities and on the SiH.

The inverse relaxation time in an epitaxial (monolayer) graphene with use of the electronic spectrum [15] in the ideal graphene sheet near $\varepsilon_{\mathrm{F}}$ is derived in the form

$$
\tau^{-1}=\tau_{i m p}^{-1}\left(1+N^{-1}(1-c) \sum_{i=0}^{N} \alpha_{i} \delta T\right) .
$$

Here $\delta=\pi R^{2} m / \hbar^{2} \approx 0.1 K^{-1}, R$ is the radius of the 1 -st coordination sphere and $\tau_{\text {imp }}^{-1} \approx 2 \cdot 10^{15} \mathrm{~s}^{-1}$.
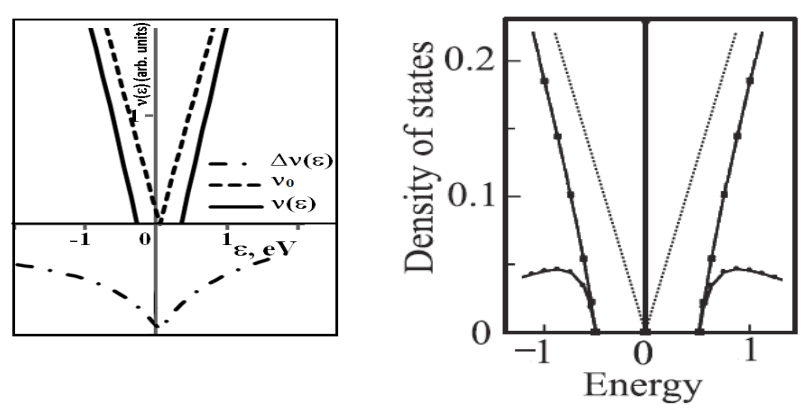

The contribution to DOS from non-ideal terms is determined by the rule [7]

$$
\Delta \nu=-\pi^{-1} \operatorname{Im} \operatorname{Tr}\left(<G>-G_{0}\right)
$$

with the averaged $G F<G>$ in an epitaxial graphene. Calculating $<G>$ we have account of the multiple elastic electron scattering on the impurities and $\mathrm{SiH}$. The result for $\Delta v$ looks as

$$
\Delta v=-\pi^{-1} \operatorname{Im} \operatorname{Tr} \Delta G=\hbar(2 \tau)^{-1} \int p d p\left[\left(\varepsilon-\varepsilon_{p}\right)^{2}+\left(\hbar(2 \tau)^{-1}\right)^{2}\right] .
$$

The resulting expression for contribution to DOS in epitaxial graphene consists of two terms:

$$
\begin{aligned}
& \Delta v=\frac{1}{\hbar^{2} v_{F}^{2}} \times \\
& \times\left[\frac{\hbar}{2 \tau} \ln \left(1+\frac{p_{0} v_{F}\left(p_{0} v_{F}-2 \varepsilon\right)}{\varepsilon^{2}+(\hbar / 2 \tau)^{2}}\right)+2 \varepsilon \operatorname{arctg}\left(\frac{p_{0} v_{F}(\hbar / 2 \tau)}{(\hbar / 2 \tau)^{2}+\left(\varepsilon-p_{0} v_{F}\right) \varepsilon}\right)\right],
\end{aligned}
$$

where $p_{F}$ is the Fermi momentum.

We analyze (15) near the Fermi level at the fixed temperature in dependence on the sign of sum of parameters of short-range order because the latter may be changed after doping and degassing of graphene. In Fig. 4a the calculated DOS in graphene is represented as a sum of 2 terms corresponding to electron scattering in ideal graphene and scattering on the $\mathrm{SiH}$. From this figure one may see that the contribution $\Delta \nu(\varepsilon)$ (the dash-dot line) calculated at $T=10 \mathrm{~K}$, for $\sum \alpha_{\mathrm{i}}=-0.6$, is negative, nonlinear and has a minimum at $\varepsilon_{\mathrm{F}}$. In an ideal graphene DOS $\nu_{0}(\varepsilon)$ (the dotted line) is linear and positive. As a result the sum of these two contributions gives a gap in curve $\nu(\varepsilon)$ (the solid line) near $\varepsilon_{\mathrm{F}}$ which is in an agreement with the data [12] presented on Fig. 4 (second figure).

In turn, on two last plots in Fig. 4 it is presented the contribution to DOS $\Delta v(\varepsilon)$ calculated at $T=1 \mathrm{~K}$,
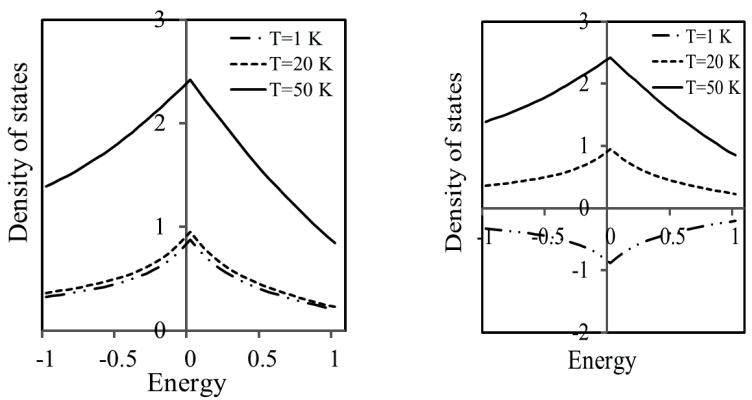

Fig. 4. a)The contribution to DOS in graphene calculated at $T=10 \mathrm{~K}$ for $\sum \alpha=-0.6$ (the dash-dot line), DOS in an ideal graphene $v_{0}(\varepsilon)$ (the dotted line) and the full DOS $v(\varepsilon)=v_{0}(\varepsilon)+\Delta v(\varepsilon)$ (the solid line). b) the data of [12]-the dotted line corresponds to DOS in ideal graphene and the solid line - to DOS in bydrogenated graphene. c) Contribution to DOS $\Delta v(\varepsilon)$ calculated for $\Sigma \alpha=0.2$ and d) for $\Sigma \alpha=-0.2$ at $T=1,20,50 \mathrm{~K}$. 

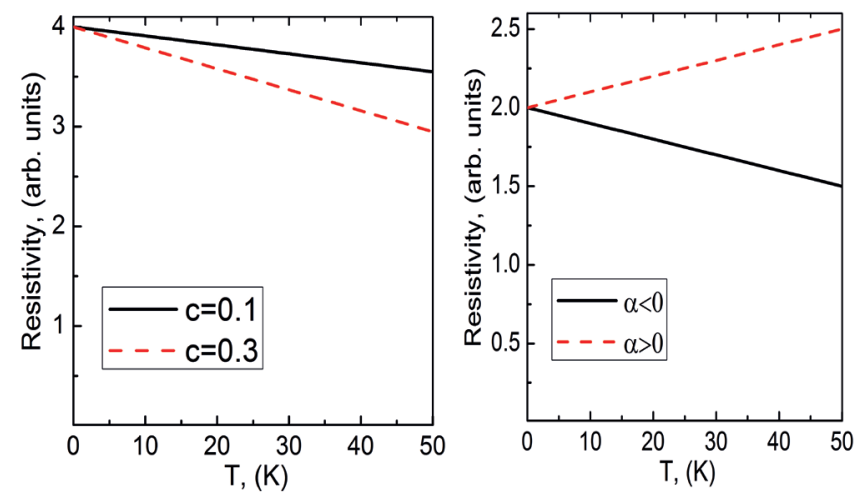

Fig. 5. Contribution to $R(T)$, found for $\alpha=-0.1$ (solid line), $\alpha=0.1$ (dashed line), $c=0.1$ (solid line), $c=0.3$.

20K, 50K. $\Delta v(\varepsilon)$ at the Fermi level increases when $T$ rises if $\Sigma \alpha>0$ and decreases if $\Sigma \alpha<0$.

Finally, the contribution to resistivity in non-ideal graphene from electron scattering by impurities and $\mathrm{SiH}$ may be also calculated similar to $[13,14]$ using electronic spectrum for graphene [15]:

$$
R(T)=m / e^{2} n\left(\tau_{i m p}^{-1}+2 \pi U_{0}^{-2} v_{0} c(1-c) \sum_{i=0}^{N} \alpha_{i} \delta T\right),
$$

where $n$ is the concentration of charge carriers. $R(T)$ consists of 2 terms corresponding to scattering on impurities and $\mathrm{SiH} . \mathrm{R}(T)$ strongly depends on $\mathrm{n}$ which is known to differ in the different types of graphene and may change a value on several orders. We present on Fig. 5 the dependence on $T$ of $R(T)$ at fixed $n$ (Fig. 5, left) and fixed sum of parameters of short-range order (Fig. 5, right).

\section{CONCLUSION}

We have studied the influence of different kinds of gaps in bilayer graphene in the 2-and 4-band models on longitudinal and Hall optical conductivities in dependence of the choice of the gaps that break the time reversal symmetry. The 2-band model is applicable for energies $E<100 \mathrm{meV}$ and the 4-band model up to energies when continuum approximation is valid with firstly obtained new form of the conductivities (8). The results (for the Faraday and Kerr angles analysis when radiation passes through the bilayer on substrates) may be applied for graphene-based optoelectronics. The resulting expressions for the contribution to DOS (15) and resistivity (16) are obtained depending on concentration of impurities and the parameters of short-range order for non-ideal graphene with metallic GF. The last result means, the change in the defect structure of graphene results in metallization of epitaxial graphene due to the increasing contribution to DOS in agreement with [12].
The results of the study show that the lowtemperature behavior of $R(T)$ and DOS is determined by the short range ordered structure of non-ideal graphene. Another issue which we elaborating is to get realistic model of $k$-layer graphene $(k=1,2)$ on substrate by replacing the metallic GF on graphene GF.

\section{ACKNOWLEDGMENTS}

The work was partially supported by the grant of Leading Scientific Schools of the Russian Federation under Project No. 88.2014.2.

\section{REFERENCES}

1. Novoselov KS, McCann E, Morozov SV, Fal'ko VI, Katsnelson MI, Zeitler U, Jiang D, Schedin F, Geim AK. Unconventional quantum Hall effect and Berry's phase of $2 \pi$ in bilayer graphene. Nat. Phys., 2006, 2(3):177-180.

2. McCann E, Fal'ko VI. Landau-Level Degeneracy and Quantum Hall Effect in a Graphite Bilayer. Phys. Rev. Lett., 2006, 96:086805.

3. McCann E, Abergel DSL, Fal'ko VI. Electrons in bilayer graphene. Solid State Commun., 2007, 143(1):110-115.

4. Falkovsky LA. Magneto-optics of monolayer and bilayer graphene. JETP Letters, 2013, 97(7):429-438.

5. Slonczewski JC, Weiss PR. Band Structure of Graphite. Phys. Rev., 1958, 109:272-279.

6. McClure JW. Band Structure of Graphite and de Haas-van Alphen Effect. Phys. Rev., 1957, 108(3):612-618.

7. Abrikosov AA, Gorkov LP, Dzyaloshinskii IE. Metody kvantovoy teorii polya $v$ statisticheskoy fizike [Methods of quantum field theory in statistical physics]. Moscow, Fizmatgiz Publ., 1962.

8. Egorushkin VE, Melnikova NV, Ponomarev AN, Reshetnyak AA. Anomalous thermal conductivity in multiwalled carbon nanotubes with impurities and short-range order. J. Physics: Conf. Series, 2010, 248(1):012005.

9. Gorbar EV, Gusynin VP, Kuzmenko AB, Sharapov SG. Magneto-Optical and Optical Probes of Gapped Ground States of Bilayer Graphene. Phys. Rev. B, 2012, 86:075414.

10. Gusynin VP, Reshetnyak AA, Sharapov SG, 2015 (in press). 
174 NADEZHDA G. BOBENKO, DALIBOR ČEVIZOVIĆ,

11. Davydov SYu. Adsorption Induced Energy Gap in the Density of States of Single Sheet Graphene. Semiconductors, 2012, 46(2):193-198.

12. Dröscher S, Roulleau P, Molitor F, Studerus P, Stampfer C, Ihn T, Enssli K. Quantum capacitance and density of states of graphene. Appl. Phys. Lett., 2010, 96, 152104.

13. Egorushkin V, Mel'nikova N, Ponomarev A, Bobenko N. Low-temperature peculiarities of electron transport properties of carbon nanotubes. J. Mat. Sci. Eng., 2011, 1(2):161-167.

14. Egorushkin VE, Melnikova NV, Ponomarev AN, Bobenko NG. Low-Temperature Thermopower in Disordered Carbon Nanotubes. Nanosystems, 2013, 4(5):622-629.

15. Wallace PR. The Band Theory of Graphite. Phys. Rev., 1947, 71(9):622-634. 